\title{
Nash and Stackelberg Equilibria for Dynamic Cheap Talk and Signaling Games*
}

\author{
Serkan Sarıtaş ${ }^{1}$, Serdar Yüksel ${ }^{2}$, and Sinan Gezici ${ }^{1}$
}

\begin{abstract}
Simultaneous (Nash) and sequential (Stackelberg) equilibria of two-player dynamic quadratic cheap talk and signaling game problems are investigated under a perfect Bayesian formulation. For the dynamic scalar and multidimensional cheap talk, the Nash equilibrium cannot be fully revealing whereas the Stackelberg equilibrium is always fully revealing. Further, the final state Nash equilibria have to be essentially quantized when the source is scalar and has a density, and non-revealing for the multi-dimensional case. In the dynamic signaling game where the transmission of a GaussMarkov source over a memoryless Gaussian channel is considered, affine policies constitute an invariant subspace under best response maps for both scalar and multi-dimensional sources under Nash equilibria; however, the Stackelberg equilibrium policies are always linear for scalar sources but may be non-linear for multi-dimensional sources. Further, under the Stackelberg setup, the conditions under which the equilibrium is non-informative are derived for scalar sources.
\end{abstract}

\section{INTRODUCTION}

Signaling games and cheap talk are concerned with a class of Bayesian games where an informed player (encoder or sender) transmits information to another player (decoder or receiver). In these problems, the objective functions of the players are not aligned unlike the ones in the classical communication problems. The cheap talk problem was studied by Crawford and Sobel [1], who obtained the surprising result that under some technical conditions on the cost functions, the cheap talk problem only admits equilibria that involve quantized encoding policies. This is in contrast with the case where the goals are aligned in classical communication and information theory.

\section{A. Literature Review}

The cheap talk and signaling game problems are applicable in networked control systems when a communication channel exists among competitive and non-cooperative decision makers [2], [3]. The reader is referred to [4] for a detailed discussion and references.

There have been extensive contributions to cheap talk and signaling games in the economics literature (see [4] and [5] for a detailed review). [6] considers the dynamic setting

*This research was supported in part by the Natural Sciences and Engineering Research Council (NSERC) of Canada, and the Scientific and Technological Research Council (TÜBİTAK) of Turkey.

${ }^{1}$ S. Sarıtaş and S. Gezici are with the Department of Electrical and Electronics Engineering, Bilkent University, 06800, Ankara, Turkey \{serkan, gezici\}@ee.bilkent.edu.tr

${ }^{2} \mathrm{~S}$. Yüksel is with the Department of Mathematics and Statistics, Queen's University, Kingston, Ontario, Canada, K7L 3N6 yuksel@mast.queensu.ca where the source is a fixed random variable distributed according to some density on $[0,1]$ (see Remark 2.2 for a detailed discussion).

Relevant papers in the control community involve [7], [8], [9], which consider Stackelberg equilibria under various setups.

This paper builds on and generalizes our earlier work in [4] and [10]. In [4], we considered static Nash and Stackelberg equilibria for more general sources than what was studied in Crawford and Sobel [1]. In [10], we investigated repeated games where the source process was an independent and identically distributed (i.i.d.) process.

\section{B. Preliminaries}

A static cheap talk problem can be formulated as follows: An informed player (encoder) knows the value of the $\mathbb{M}$ valued random variable $M$ and transmits the $\mathbb{X}$-valued random variable $X$ to another player (decoder), who generates his/her $\mathbb{M}$-valued optimal decision $U$ upon receiving $X$. The policies of the encoder and decoder are assumed to be deterministic; i.e., $x=\gamma^{e}(m)$ and $u=\gamma^{d}(x)=\gamma^{d}\left(\gamma^{e}(m)\right)$. The encoder's goal is to minimize

$$
J^{e}\left(\gamma^{e}, \gamma^{d}\right)=\mathbb{E}\left[c^{e}(m, u)\right]
$$

whereas, the decoder's goal is to minimize

$$
J^{d}\left(\gamma^{e}, \gamma^{d}\right)=\mathbb{E}\left[c^{d}(m, u)\right]
$$

by finding optimal policies $\gamma^{e}$ and $\gamma^{d}$, respectively. If the transmitted signal $x$ is also an explicit part of the cost function $c^{e}$ or $c^{d}$, then the communication between the players is not costless and the formulation turns into a signaling game problem. Such problems are studied under the tools and concepts provided by game theory since the goals are not aligned. In the simultaneous game-play; i.e., the encoder and decoder announce their policies at the same time, a pair of policies $\left(\gamma^{*, e}, \gamma^{*, d}\right)$ is said to be a (simultaneous) Nash equilibrium if

$$
\begin{array}{ll}
J^{e}\left(\gamma^{*, e}, \gamma^{*, d}\right) \leq J^{e}\left(\gamma^{e}, \gamma^{*, d}\right) & \forall \gamma^{e} \in \Gamma^{e} \\
J^{d}\left(\gamma^{*, e}, \gamma^{*, d}\right) \leq J^{d}\left(\gamma^{*, e}, \gamma^{d}\right) & \forall \gamma^{d} \in \Gamma^{d}
\end{array}
$$

where $\Gamma^{e}$ and $\Gamma^{d}$ are the sets of all deterministic functions from $\mathbb{M}$ to $\mathbb{X}$ and from $\mathbb{X}$ to $\mathbb{M}$, respectively. Similarly, in the sequential game-play; i.e., first the encoder announces his/her policy, then the decoder (accordingly) announces 
his/her policy, a pair of policies $\left(\gamma^{*, e}, \gamma^{*, d}\right)$ is said to be a Stackelberg equilibrium if

$$
\begin{aligned}
& J^{e}\left(\gamma^{*, e}, \gamma^{*, d}\left(\gamma^{*, e}\right)\right) \leq J^{e}\left(\gamma^{e}, \gamma^{*, d}\left(\gamma^{e}\right)\right) \quad \forall \gamma^{e} \in \Gamma^{e} \\
& \text { where } \gamma^{*, d}\left(\gamma^{e}\right) \text { satisfies } \\
& J^{d}\left(\gamma^{e}, \gamma^{*, d}\left(\gamma^{e}\right)\right) \leq J^{d}\left(\gamma^{e}, \gamma^{d}\left(\gamma^{e}\right)\right) \quad \forall \gamma^{d} \in \Gamma^{d} .
\end{aligned}
$$

If an equilibrium is achieved when $\gamma^{*, e}$ is non-informative (e.g., the transmitted message and the source are independent) and $\gamma^{*, d}$ uses only the prior information (since the received message is useless), then we call such an equilibrium a non-informative (babbling) equilibrium. The following is a useful observation, which follows from [1]:

Proposition 1.1: A non-informative (babbling) equilibrium always exists for the cheap talk game.

Heretofore, only static (one-stage) games are considered. If a game is played over a number of time periods, the game is called a dynamic game. Let $m_{[0, N-1]}=$ $\left\{m_{0}, m_{1}, \cdots, m_{N-1}\right\}$ be a collection of random variables to be encoded sequentially (causally) to a decoder. In the $k$-th stage of an $N$-stage game, the encoder transmits $x_{k}=\gamma_{k}^{e}\left(\mathcal{I}_{k}^{e}\right)$ to the decoder who generates his/her optimal decision $u_{k}=\gamma_{k}^{d}\left(\mathcal{I}_{k}^{d}\right)$ where $\mathcal{I}_{k}^{e}=\left\{m_{[0, k]}, x_{[0, k-1]}\right\}$ and $\mathcal{I}_{k}^{d}=\left\{x_{[0, k]}\right\}$ with $\mathcal{I}_{0}^{e}=\left\{m_{0}\right\}$. The encoder's goal is to minimize

$$
J^{e}\left(\gamma^{e}, \gamma^{d}\right)=\mathbb{E}\left[\sum_{k=0}^{N-1} c_{k}^{e}\left(m_{k}, u_{k}\right)\right],
$$

whereas, the decoder's goal is to minimize

$$
J^{d}\left(\gamma^{e}, \gamma^{d}\right)=\mathbb{E}\left[\sum_{k=0}^{N-1} c_{k}^{d}\left(m_{k}, u_{k}\right)\right]
$$

by finding the optimal policy sequences $\gamma_{[0, N-1]}^{e}=$ $\left\{\gamma_{0}^{e}, \gamma_{1}^{e}, \cdots, \gamma_{N-1}^{e}\right\}$ and $\gamma_{[0, N-1]}^{d}=\left\{\gamma_{0}^{d}, \gamma_{1}^{d}, \cdots, \gamma_{N-1}^{d}\right\}$, respectively. In this study, the quadratic cost functions are assumed; i.e., $c_{k}^{e}\left(m_{k}, u_{k}\right)=\left(m_{k}-u_{k}-b\right)^{2}$ and $c_{k}^{d}\left(m_{k}, u_{k}\right)=\left(m_{k}-u_{k}\right)^{2}$ where $b$ is the bias term as in [1] and [4].

Under both equilibria concepts, we consider the setups where the decision makers act optimally for each history path of the game (available to each decision maker) and the updates are Bayesian, and thus the equilibria are to be interpreted under a perfect Bayesian equilibria concept. Since we assume such a (perfect Bayesian) framework, the equilibria lead to sub-game perfection and each decision maker makes optimal Bayesian decisions for every realized play path.

\section{Contributions}

This study focuses on the multi-stage setup of a cheap talk problem introduced by Crawford and Sobel [1]. We extend the static cheap talk and signaling game studied in our previous work [4] to a dynamic setup, and extend the analysis in [10] from i.i.d. and scalar sources to Markov and multi-dimensional sources. The main contributions of this paper can be summarized as follows:

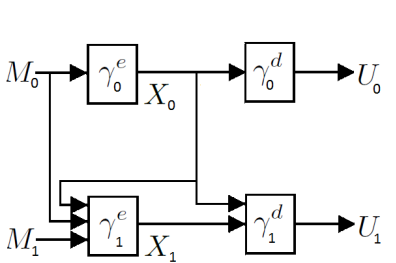

(a) 2-stage cheap talk.

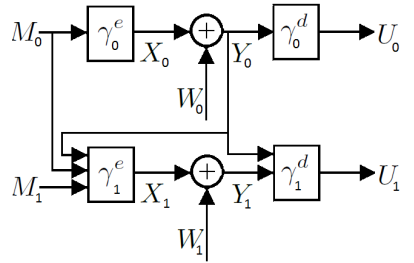

(b) 2-stage signaling game.
Fig. 1: General system model

- We prove that, in the dynamic cheap talk game, under Nash equilibria, the last stage equilibria are quantized for a Markov source with arbitrary conditional probability measure with a density, and fully revealing equilibria cannot exist in general (see Remark 2.1).

- We show that the equilibria are fully revealing in the dynamic multi-dimensional cheap talk under Stackelberg equilibria whereas the equilibrium cannot be fully revealing under Nash equilibria.

- We show that affine policies constitute an invariant subspace under best response maps under Nash equilibria for the dynamic multi-dimensional signaling game.

- Dynamic Stackelberg signaling equilibria for scalar Gauss-Markov sources and scalar Gaussian channels are always linear, which is not necessarily the case for multi-dimensional setups. Further, the conditions for the existence of informative equilibria are provided for scalar sources by using information theoretic arguments.

\section{Dynamic Cheap Talk for Markov Sources}

For the purpose of illustration, the system model of the 2-stage dynamic cheap talk is depicted in Fig. 1-(a).

\section{A. A Supporting Result : A Static Scalar Cheap Talk with Randomized Policies}

To facilitate our analysis to handle certain intricacies that arise due to the dynamic setup in the paper, and also to present an independently interesting result in itself, in the following we state that the result in [4, Theorem 3.2] also holds when the encoder is allowed to adapt randomized encoding policies by extending [1, Lemma 1] as follows:

Theorem 2.1: The conclusion of [4, Theorem 3.2], i.e., that an equilibrium policy is equivalent to a quantized policy, also holds if the policy space of the encoder is extended to the set of all stochastic kernels from $\mathbb{M}$ to $\mathbb{X}$ for any arbitrary source that admits a density. That is, even when the encoder is allowed to use private randomization, all equilibria are equivalent to those that are attained by quantized equilibria.

Proof: [1, Lemma 1] proves that all equilibria have finitely many partitions when the source has a bounded support. [4, Theorem 3.2] extends this result to a countable number of partitions for deterministic equilibria for any source with an arbitrary probability measure. The result 
follows by utilizing [4, Theorem 3.2] and [1, Lemma 1].

Theorem 2.1 will be used crucially in the following analysis; since in a dynamic game, at a given time stage, the source variables from the earlier stages can serve as private randomness for the encoder.

\section{B. Repeated i.i.d. Scalar Games: Nash Equilibria}

We first review our previous results on dynamic signaling games [10] where the source was assumed to be i.i.d.

Theorem 2.2: [10] In the $N$-stage repeated cheap talk game, the equilibrium policies for the final stage must be quantized almost surely for any collection of policies $\left(\gamma_{[0, N-2]}^{e}, \gamma_{[0, N-2]}^{d}\right)$ and for any real-valued source model with arbitrary probability measure $P\left(\mathrm{~d} m_{N-1}\right)$ which admits a density. If the source $m_{N-1}$ has a bounded support, the first $N-1$ stages cannot have fully revealing equilibria concurrently.

Remark 2.1: [10] The boundedness assumption for the support of the measure $P\left(\mathrm{~d} m_{N-1}\right)$ can be relaxed for Theorem 2.2. In particular, a source with a probability measure $P\left(\mathrm{~d} m_{N-1}\right)$ that results in finitely many quantization bins in a static Nash equilibrium satisfies the statements; e.g., when the source admits an exponentially distributed real random variable (see [11]).

\section{Dynamic Game with a Markov Source: Nash Equilibria}

In this part, the source $M_{k}$ is assumed to be real valued Markovian for $k=0,1, \ldots, N-1$. The following result generalizes Theorem 2.2, which only considered i.i.d. sources.

Theorem 2.3: In the $N$-stage dynamic cheap talk game with a Markov source, the equilibrium policies for the final stage must be quantized almost surely for any collection of policies $\left(\gamma_{[0, N-2]}^{e}, \gamma_{[0, N-2]}^{d}\right)$ and for any real-valued source model with arbitrary conditional probability measure $P\left(\mathrm{~d} m_{N-1} \mid m_{N-2}\right)$ which admits a density almost surely.

Proof: Here, we prove the results for the 2-stage games, the extension is merely technical. The expected cost of the second stage encoder $J_{1}^{e}$ can be written as

$$
\begin{aligned}
J_{1}^{e}= & \int p\left(\mathrm{~d} m_{0}, \mathrm{~d} m_{1}, \mathrm{~d} x_{0}, \mathrm{~d} x_{1}\right) c_{1}^{e}\left(m_{1}, u_{1}\right) \\
= & \int p\left(\mathrm{~d} x_{0}\right) \int p\left(\mathrm{~d} m_{1} \mid x_{0}\right) p\left(\mathrm{~d} m_{0} \mid m_{1}, x_{0}\right) \\
& \times c_{1}^{e}\left(m_{1}, \gamma_{1}^{d}\left(x_{0}, \gamma_{1}^{e}\left(m_{0}, m_{1}, x_{0}\right)\right)\right)
\end{aligned}
$$

The inner integral of (3) can be considered as an expression for a given $x_{0}$. Thus, given the second stage encoder and decoder policies $\gamma_{1}^{e}\left(m_{0}, m_{1}, x_{0}\right)$ and $\gamma_{1}^{d}\left(x_{0}, x_{1}\right)$, it is possible to define policies which are parametrized by the common information $x_{0}$ almost surely so that $\widehat{\gamma}_{x_{0}}^{e}\left(m_{0}, m_{1}\right)=$ $\gamma_{1}^{e}\left(m_{0}, m_{1}, x_{0}\right)$ and $\widehat{\gamma}_{x_{0}}^{d}\left(x_{1}\right)=\gamma_{1}^{d}\left(x_{0}, x_{1}\right)$. After following similar arguments to those in the proof of Theorem 2.2, the second stage encoder policy becomes $\widehat{\gamma}_{x_{0}}^{e}\left(m_{0}, m_{1}\right) \stackrel{(a)}{=}$ $\widehat{\gamma}_{x_{0}}^{e}\left(g\left(m_{1}, r\right), m_{1}\right)=\widetilde{\gamma}_{x_{0}}^{e}\left(m_{1}, r\right)$ where (a) holds since any stochastic kernel from a complete, separable and metric space to another one, $P\left(\mathrm{~d} m_{0} \mid m_{1}\right)$, can be realized by some measurable function $m_{0}=f\left(m_{1}, r\right)$ where $r$ is a $[0,1]$ valued independent random variable (see Lemma 1.2 in [12], or Lemma 3.1 in [13]). Hence, the equilibria are quantized almost surely by Theorem 2.2.

Remark 2.2: A related setup has been studied in [6] where it has been shown that there can indeed be a fully revealing equilibrium if an individual source is transmitted repeatedly (thus the Markov source is a constant source). We note that there is no contradiction since for such a source, equilibria can be carefully constructed so that even a quantized final stage equilibrium can be made to be fully revealing.

\section{Dynamic Cheap Talk under Stackelberg Equilibria}

The equilibrium drastically changes under a Stackelberg formulation.

Theorem 2.4: [10, Theorem 3.3] An equilibrium has to be fully revealing in the dynamic Stackelberg cheap talk game regardless of the source model.

Proof: Since the decoder is myopic, the optimal decoder actions are $u_{k}^{*}=\mathbb{E}\left[m_{k} \mid x_{[0, k]}\right]$ for $k=0,1, \ldots, N-$ 1. Then the total encoder cost becomes $J^{e}\left(\gamma^{e}, \gamma^{d}\right)=$ $\mathbb{E}\left[\sum_{k=0}^{N-1}\left(m_{k}-u_{k}\right)^{2}\right]+N b^{2}$, which effectively reduces the game setup to a team setup, resulting in fully informative equilibria.

\section{E. Dynamic Multi-Dimensional Cheap Talk}

In this part, Nash and Stackelberg equilibria of the dynamic multi-dimensional cheap talk are analyzed in sequence.

Since there may be discrete, non-discrete or even linear Nash equilibria in the static (one-stage) multi-dimensional cheap talk by [4, Theorem 3.4], the equilibrium policies are hard to characterize; however, we still have the following:

Theorem 2.5: The Nash equilibrium cannot be fully revealing in the static (one-stage) multi-dimensional cheap talk when the source has positive measure for every nonempty open set almost surely.

Proof: Let there be an equilibrium, and define two actions of the decoder as $\vec{u}^{\alpha}$ and $\vec{u}^{\beta}$. By following the similar approach to that in the proof of [4, Theorem 3.2], it can be deduced that the length of $\vec{b}$ along the $\vec{d} \triangleq \vec{u}^{\beta}-\vec{u}^{\alpha}$ direction should not exceed half of the distance between $\vec{u}^{\alpha}$ and $\vec{u}^{\beta}$; i.e., $\|\vec{b} \vec{d}\| \leq\|\vec{d}\| / 2$, where $\vec{b}_{\vec{d}}$ is the projection of $\vec{b}$ along the direction of $\vec{d}$. Since $\vec{d}$ can be any vector at a fully revealing equilibrium by the assumption on the source, $\|\vec{b} \vec{d}\| \leq\|\vec{d}\| / 2$ cannot be satisfied unless $\vec{b}=\overrightarrow{0}$. Thus, there cannot be a fully revealing equilibrium in the static multidimensional cheap talk.

We can extend this result to the dynamic multidimensional cheap talk as follows:

Theorem 2.6: The final stage Nash equilibria cannot be fully revealing in the dynamic multi-dimensional cheap talk for i.i.d. sources and Markov sources when the conditional distribution $P\left(\mathrm{~d} \vec{m}_{N-1} \mid \vec{m}_{N-2}\right)$ has positive measure for every non-empty open set almost surely. 
Unlike the different characteristics between Nash equilibria of the dynamic scalar and multi-dimensional cheap talk, fully revealing characteristics of the Stackelberg equilibrium still hold for the dynamic multi-dimensional cheap talk, as for the scalar case:

Theorem 2.7: The Stackelberg equilibria in the dynamic multi-dimensional cheap talk can be obtained by extending its scalar case; i.e., it is unique and corresponds to a fully revealing encoder policy as in the scalar case.

Proof: Similar to the scalar case in Theorem 2.4, the optimal decoder actions are $\vec{u}_{k}^{*}=\mathbb{E}\left[\vec{m}_{k} \mid \vec{x}_{[0, k]}\right]$ for $k=0,1, \ldots, N-1$. Then the total encoder cost becomes $J^{e}\left(\gamma^{e}, \gamma^{d}\right)=\mathbb{E}\left[\sum_{k=0}^{N-1}\left\|\vec{m}_{k}-\vec{u}_{k}\right\|^{2}\right]+N\|\vec{b}\|^{2}$, which effectively reduces the game setup to a team setup, resulting in fully informative equilibria.

\section{DYNAMIC QUADRATIC GAUSSIAN SignALING GAMES FOR SCALAR GAUSS-MARKOV SOURCES}

The dynamic signaling game setup is similar to the dynamic cheap talk setup except that there exists an additive Gaussian noise channel between the encoder and decoder at each stage, and the encoder has a soft power constraint. For the purpose of illustration, the system model of the 2-stage dynamic signaling game is depicted in Fig. 1-(b).

Here, the source is assumed to be a Markov source with initial Gaussian distribution; i.e. $M_{0} \sim \mathcal{N}\left(0, \sigma_{M_{0}}^{2}\right)$ and $M_{k+1}=g M_{k}+V_{k}$ where $g \in \mathbb{R}$ and $V_{k} \sim \mathcal{N}\left(0, \sigma_{V_{k}}^{2}\right)$ is an i.i.d. Gaussian noise sequence for $k=0,1, \ldots, N-$ 2 . The channels between the encoder and the decoder are assumed to be i.i.d. additive Gaussian channels; i.e. $W_{k} \sim \mathcal{N}\left(0, \sigma_{W_{k}}^{2}\right)$, and $W_{k}$ and $V_{l}$ are independent for $k=0,1, \ldots, N-1$ and $l=0,1, \ldots, N-2$. In the $k$ th stage of the $N$-stage game, the information available at the encoder and the decoder is $\mathcal{I}_{k}^{e}=\left\{m_{[0, k]}, y_{[0, k-1]}\right\}$ (a noiseless feedback channel is assumed) and $\mathcal{I}_{k}^{d}=\left\{y_{[0, k]}\right\}$ with $y_{k}=x_{k}+w_{k}$, respectively. The encoder's goal is to minimize

$$
J^{e}\left(\gamma^{e}, \gamma^{d}\right)=\mathbb{E}\left[\sum_{k=0}^{N-1} c_{k}^{e}\left(m_{k}, x_{k}, u_{k}\right)\right]
$$

whereas, the decoder's goal is to minimize

$$
J^{d}\left(\gamma^{e}, \gamma^{d}\right)=\mathbb{E}\left[\sum_{k=0}^{N-1} c_{k}^{d}\left(m_{k}, u_{k}\right)\right]
$$

by finding the optimal policy sequences $\gamma_{[0, N-1]}^{e}$ and $\gamma_{[0, N-1]}^{d}$, respectively. The cost functions are modified as $c_{k}^{e}\left(m_{k}, x_{k}, u_{k}\right)=\left(m_{k}-u_{k}-b\right)^{2}+\lambda x_{k}^{2}$ and $c_{k}^{d}\left(m_{k}, u_{k}\right)=$ $\left(m_{k}-u_{k}\right)^{2}$. Note that a power constraint with an associated multiplier is appended to the cost function of the encoder, which corresponds to power limitation for transmitters in practice.

\section{A. Dynamic Nash Equilibria for Scalar Gauss-Markov Sources}

In dynamic signaling games, affine policies constitute an invariant subspace under best response maps for Nash equilibria, as stated in [10, Theorem 4.2]:

Theorem 3.1: [10, Theorem 4.2]

i) If the encoder uses affine policies at all stages, then the decoder will also be affine at all stages.

ii) If the decoder uses affine policies at all stages, then the encoder will also be affine at all stages.

\section{B. Dynamic Stackelberg Equilibria for Scalar Gauss- Markov Sources}

The equilibrium drastically changes under the Stackelberg assumption.

Theorem 3.2: An equilibrium has to be always linear in the dynamic Stackelberg signaling game. Furthermore, there does not exist an informative (affine or non-linear) equilibrium in the $N$-stage dynamic scalar signaling game under the Stackelberg assumption; i.e., the only equilibrium is the non-informative one, if $\lambda \geq \max _{k=0,1, \ldots, N-1} \frac{\sigma_{M_{k}}^{2}}{\sigma_{W_{k}}^{2}} \sum_{i=0}^{N-k-1} g^{2 i}$.

Proof Sketch: First part is due to [10, Theorem 4.1]. For the second part, the lower bound for the encoder cost will be obtained. From the chain rule, $I\left(m_{k} ; y_{[0, k]}\right)=I\left(m_{k} ; y_{[0, k-1]}\right)+I\left(m_{k} ; y_{k} \mid y_{[0, k-1]}\right)$. By following similar arguments to those [14], [15, Theorem 11.3.1], $I\left(m_{k} ; y_{k} \mid y_{[0, k-1]}\right) \leq \frac{1}{2} \log _{2}\left(1+\frac{P_{k}}{\sigma_{W_{k}}^{2}}\right) \triangleq \widehat{C}_{k}$ where $P_{k}=\mathbb{E}\left[x_{k}^{2}\right]$. By using the orthogonality between $m_{k}-\mathbb{E}\left[m_{k} \mid m_{k-1}\right]$ and $\left(m_{k-1}, y_{[0, k-1]}\right)$, it follows that

$$
\begin{gathered}
\mathbb{E}\left[\left(m_{k}-\mathbb{E}\left[m_{k} \mid y_{[0, k-1]}\right]\right)^{2}\right]=\mathbb{E}\left[\left(m_{k}-\mathbb{E}\left[m_{k} \mid m_{k-1}\right]\right)^{2}\right] \\
+\mathbb{E}\left[\left(\mathbb{E}\left[m_{k} \mid m_{k-1}\right]-\mathbb{E}\left[m_{k} \mid y_{[0, k-1]}\right]\right)^{2}\right] \\
\stackrel{(a)}{=} \sigma_{V_{k-1}}^{2}+g^{2} \mathbb{E}\left[\left(m_{k-1}-\mathbb{E}\left[m_{k-1} \mid y_{[0, k-1]}\right]\right)^{2}\right] \\
\stackrel{(b)}{\geq} \sigma_{V_{k-1}}^{2}+g^{2} \sigma_{M_{k-1}}^{2} 2^{-2 C_{k-1}} \triangleq \sigma_{M_{k}}^{2} 2^{-2 \widetilde{C}_{k}} .
\end{gathered}
$$

Here, (a) is obtained by the iterated expectation rule, the Markov chain property, and $\mathbb{E}\left[m_{k} \mid m_{k-1}\right]=$ $\mathbb{E}\left[g m_{k-1}+v_{k-1} \mid m_{k-1}\right]=g m_{k-1}$, and (b) holds due to [15, Lemma 11.3.1]. From [15, Lemma 11.3.2], $I\left(m_{k} ; y_{[0, k-1]}\right)$ is maximized with linear policies, and the lower bound of (4) is achievable through linear policies where $\sup I\left(m_{k} ; y_{[0, k-1]}\right) \triangleq \widetilde{C}_{k}=$ $\frac{1}{2} \log _{2}\left(\frac{\sigma_{M_{k}}^{2}}{\sigma_{V_{k-1}}^{2}+g^{2} \sigma_{M_{k-1}}^{2} 2^{-2 C_{k-1}}}\right)$. Thus, we have the following recursion on upper bounds on mutual information for the $N$-stage dynamic signaling game:

$$
\begin{array}{r}
C_{k} \triangleq \sup I\left(m_{k} ; y_{[0, k]}\right)=\widehat{C}_{k}+\widetilde{C}_{k}=\frac{1}{2} \log _{2}\left(1+\frac{P_{k}}{\sigma_{W_{k}}^{2}}\right) \\
+\frac{1}{2} \log _{2}\left(\frac{\sigma_{M_{k}}^{2}}{\sigma_{V_{k-1}}^{2}+g^{2} \sigma_{M_{k-1}}^{2} 2^{-2 C_{k-1}}}\right)
\end{array}
$$


for $k=1,2, \ldots, N-1$ with $C_{0}=\frac{1}{2} \log _{2}\left(1+\frac{P_{0}}{\sigma_{W_{0}}^{2}}\right)$. Let the lower bound of $\mathbb{E}\left[\left(m_{k}-\mathbb{E}\left[m_{k} \mid y_{[0, k]}\right]\right)^{2}\right]$ be $\Delta_{k}$; i.e., $\mathbb{E}\left[\left(m_{k}-\mathbb{E}\left[m_{k} \mid y_{[0, k]}\right]\right)^{2}\right] \geq \sigma_{M_{k}}^{2} 2^{-2 C_{k} \triangleq \Delta_{k}}$. Then the following recursion can be obtained for the $N$-stage dynamic signaling game:

$$
\Delta_{k}=\frac{\sigma_{V_{k-1}}^{2}+g^{2} \Delta_{k-1}}{1+\frac{P_{k}}{\sigma_{W_{k}}^{2}}} \text { for } k=1,2, \ldots, N-1
$$

with $\Delta_{0}=\frac{\sigma_{M_{0}}^{2}}{1+\frac{P_{0}}{\sigma_{W_{0}}^{2}}}$. In an equilibrium, since the decoder always chooses $u_{k}=\mathbb{E}\left[m_{k} \mid y_{[0, k]}\right]$ for $k=0,1, \ldots, N-1$, the total encoder cost for the first stage can be lower bounded by $J_{0}^{e, l o w e r}=\sum_{i=0}^{N-1}\left(\Delta_{i}+\lambda P_{i}+b^{2}\right)$. Now observe the following:

$$
\frac{\partial \Delta_{l}}{\partial P_{k}}= \begin{cases}g^{2}\left(1+\frac{P_{l}}{\sigma_{W_{l}}^{2}}\right)^{-1} \frac{\partial \Delta_{l-1}}{\partial P_{k}}-\frac{1}{\sigma_{W_{l}}^{2}} \frac{\partial P_{l}}{\partial P_{k}} & \text { if } l<k \\ \times\left(\sigma_{V_{l-1}}^{2}+g^{2} \Delta_{l-1}\right)\left(1+\frac{P_{l}}{\sigma_{W_{l}}^{2}}\right)^{-2} & \text { if } l \geq k\end{cases}
$$

where $\frac{\partial P_{l}}{\partial P_{k}}=0$ for $l<k$ due to the information structure of the encoder. Then we obtain $\frac{\partial J_{0}^{e, l o w e r}}{\partial P_{N-1}} \geq \lambda-\frac{\sigma_{M_{N-1}}^{2}}{\sigma_{W_{N-1}}^{2}}$ (see [5] for the derivation). If $\lambda>\frac{\sigma_{M_{N-1}}^{2}}{\sigma_{W_{N-1}}^{2}}$, then $\frac{\partial J_{0}^{e, \text { lower }}}{\partial P_{N-1}}>$ 0 , which implies that $J_{0}^{e, l o w e r}$ is an increasing function of $P_{N-1}$. For this case, in order to minimize $J_{0}^{e, l o w e r}$, $P_{N-1}$ must be chosen as 0 ; i.e., $P_{N-1}^{*}=0$. Then, after applying the similar approach and the backward induction (see [5] for a detailed proof), it can be deduced that if $\lambda>\max _{k=0,1, \ldots, N-1} \frac{\sigma_{M_{k}}^{2}}{\sigma_{W_{k}}^{2}} \sum_{i=0}^{N-k-1} g^{2 i}$, then the lower bound $J_{0}^{e, \text { lower }}$ of the encoder costs $J_{0}^{e}$ is minimized by choosing $P_{0}^{*}=P_{1}^{*}=\cdots=P_{N-1}^{*}=0$; that is, the encoder does not signal any output. Hence, the encoder engages in a non-informative equilibrium.

\section{DYNAMIC QuAdRATIC SIGNALING GAMES FOR Multi-Dimensional GaUss-Markov SourCES}

In this section, the scalar setup is extended to the $n$ dimensional setup. Namely, $n \times n$ matrix $G$ is defined as the equivalent of the scalar $g$ in Section III, and the cost functions are $c^{e}\left(\vec{m}_{k}, \vec{x}_{k}, \vec{u}_{k}\right)=\left\|\vec{m}_{k}-\vec{u}_{k}-\vec{b}\right\|^{2}+\lambda\left\|\vec{x}_{k}\right\|^{2}$ and $c^{d}\left(\vec{m}_{k}, \vec{u}_{k}\right)=\left\|\vec{m}_{k}-\vec{u}_{k}\right\|^{2}$ where the lengths of the vectors are defined in $L_{2}$ norm and $\vec{b}$ is the bias vector.

\section{A. Dynamic Nash Equilibria for Vector Gauss-Markov Sources}

Similar to the scalar source case, affine policies constitute an invariant subspace under the best response maps for Nash equilibria when the source is multi-dimensional in the dynamic signaling games as shown below:
Theorem 4.1: i) If the encoder uses affine policies at all stages, then the decoder will be affine at all stages.

ii) If the decoder uses affine policies at all stages, then the encoder will be affine at all stages.

Proof:

i) The result is immediate through the MMSE properties for Gaussian variables.

ii) Here, the proof is presented for the 2-stage game which can be extended to the $N$-stage game. Let the decoder policies be $\overrightarrow{u_{0}}=\gamma_{0}^{d}\left(\overrightarrow{y_{0}}\right)=K \overrightarrow{y_{0}}+\vec{L}$ and $\overrightarrow{u_{1}}=\gamma_{1}^{d}\left(\overrightarrow{y_{0}}, \overrightarrow{y_{1}}\right)=M_{0} \overrightarrow{y_{0}}+M_{1} \overrightarrow{y_{1}}+\vec{N}$ where $K, M_{0}$ and $M_{1}$ are $n \times n$ matrices and $\vec{L}$ and $\vec{N}$ are $n \times 1$ vectors. Then, by a dynamic programming approach, the second stage encoder cost can be written as

$$
\begin{aligned}
J_{1}^{*, e}= & \min _{\overrightarrow{x_{1}}=\gamma_{1}^{e}\left(\overrightarrow{m_{0}}, \overrightarrow{m_{1}}, \overrightarrow{y_{0}}\right)} \mathbb{E}\left[\left\|\overrightarrow{m_{1}}-\overrightarrow{u_{1}}-\vec{b}\right\|^{2}+\lambda\left\|\overrightarrow{x_{1}}\right\|^{2}\right] \\
= & \min _{\overrightarrow{x_{1}}} \mathbb{E}\left[\left(\Lambda \overrightarrow{x_{1}}-M_{1}^{T} \Xi\right)^{T} \Lambda^{-1}\left(\Lambda \overrightarrow{x_{1}}-M_{1}^{T} \Xi\right)\right. \\
& \left.+\Xi^{T}\left(I-M_{1} \Lambda^{-1} M_{1}^{T}\right) \Xi+{\overrightarrow{w_{1}}}^{T} M_{1}^{T} M_{1} \overrightarrow{w_{1}}\right]
\end{aligned}
$$

where $\Lambda \triangleq M_{1}^{T} M_{1}+\lambda I$ and $\Xi \triangleq \overrightarrow{m_{1}}-M_{0} \overrightarrow{y_{0}}-\vec{N}-\vec{b}$. Hence, the optimal $\gamma_{1}^{e}\left(\vec{m}_{0}, \vec{m}_{1}, \overrightarrow{y_{0}}\right)$ can be chosen as

$$
\gamma_{1}^{*, e}\left(\overrightarrow{m_{0}}, \overrightarrow{m_{1}}, \overrightarrow{y_{0}}\right)=\Lambda^{-1} M_{1}^{T}\left(\vec{m}_{1}-M_{0} \overrightarrow{y_{0}}-\vec{N}-\vec{b}\right),
$$

and the minimum second stage encoder cost becomes $J_{1}^{*, e}=\mathbb{E}\left[\Xi^{T}\left(I-M_{1} \Lambda^{-1} M_{1}^{T}\right) \Xi+{\overrightarrow{w_{1}}}^{T} M_{1}^{T} M_{1} \vec{w}_{1}\right]$.

Then, by a dynamic programming approach and completing the square, the total cost of the encoder can be written as

$$
\begin{aligned}
& J_{0}^{*, e}= \min _{\overrightarrow{x_{0}}=\gamma_{0}^{e}\left(m_{0}\right)} \mathbb{E}\left[\left\|\overrightarrow{m_{0}}-\overrightarrow{u_{0}}-\vec{b}\right\|^{2}+\lambda\left\|\overrightarrow{x_{0}}\right\|^{2}+J_{1}^{*, e}\right] \\
&=\min _{\overrightarrow{x_{0}}} \mathbb{E}\left[\left(\Upsilon \overrightarrow{x_{0}}-M_{0}^{T} \Omega \Psi-K^{T} \zeta\right)^{T} \Upsilon^{-1}\right. \\
& \times\left(\Upsilon x_{0}-M_{0}^{T} \Omega \Psi-K^{T} \zeta\right)+\zeta^{T} \zeta+\Psi^{T} \Omega \Psi \\
&-\left(M_{0}^{T} \Omega \Psi+K^{T} \zeta\right)^{T} \Upsilon^{-1}\left(M_{0}^{T} \Omega \Psi+K^{T} \zeta\right) \\
&+{\overrightarrow{w_{0}}}^{T} K^{T} K \overrightarrow{w_{0}}+{\overrightarrow{v_{0}}}^{T} \Omega \overrightarrow{v_{0}}+{\overrightarrow{w_{0}}}^{T} M_{0}^{T} \Omega M_{0} \overrightarrow{w_{0}} \\
&+{\overrightarrow{w_{1}}}^{T} M_{1}^{T} M_{1}{\overrightarrow{w_{1}}}^{T}
\end{aligned}
$$

where $\Upsilon \triangleq K^{T} K+\lambda I+M_{0}^{T} \Omega M_{0}, \Omega \triangleq(I-$ $\left.M_{1}\left(M_{1}^{T} M_{1}+\lambda I\right)^{-1} M_{1}^{T}\right), \Psi \triangleq A \vec{m}_{0}-\vec{N}-\vec{b}$, and $\zeta \triangleq \vec{m}_{0}-\vec{L}-\vec{b}$. Hence, the optimal $\gamma_{0}^{e}\left(\vec{m}_{0}\right)$ is

$$
\begin{aligned}
\gamma_{0}^{*, e}\left(\vec{m}_{0}\right)=\Upsilon^{-1} & \left(\left(M_{0}^{T} \Omega A+K\right) \vec{m}_{0}\right. \\
& \left.-M_{0}^{T} \Omega(\vec{N}+\vec{b})-K^{T}(\vec{L}+\vec{b})\right) .
\end{aligned}
$$

\section{B. Dynamic Stackelberg Equilibria for Vector Gauss- Markov Sources}

Even when the encoder and the decoder have identical (non-biased) quadratic cost functions, when the source and the channel are multi-dimensional, linear policies may not be optimal; see [15, Chapter 11] for a detailed discussion. 
In particular, except for settings where matching between the source and the channel exists (building on [16], [17]), the optimality of linear policies is quite rare [18]. Matching essentially requires that the capacity achieving source probabilities and the rate-distortion achieving channel probabilistic characteristics are simultaneously realized for a given system; this is precisely the case for a scalar Gaussian source transmitted over a scalar additive Gaussian channel. One special case where such a matching holds is the case when the noise and signal power levels are identical in every channel and the distortion criterion is identical for all scalar components [19]. For further discussions on multidimensional Gaussian source and channel pairs, we refer the reader to [17]-[24].

It is evident from Theorem 4.1 that when the encoder is linear, the optimal decoder is linear. In this case, a relevant problem is to find the optimal Stackelberg policy among the linear or affine class. We refer the reader to [24][27] for a study of such problems. In particular, a dynamic programming approach can be adapted to find Stackelberg equilibria as in [28, Theorem 3] (see also [29]) when the encoder is restricted to be linear and memoryless.

\section{Concluding Remarks}

In this paper, Nash and Stackelberg equilibria for dynamic quadratic cheap talk and signaling games have been analyzed. For the dynamic cheap talk problem, we have shown that the last stage Nash equilibria are quantized for any scalar source with an arbitrary distribution which admits a density, and fully revealing Nash equilibria cannot exist in general (see Remark 2.1); whereas, the Stackelberg equilibria must be fully revealing regardless of the source model. We have also proved that the equilibria are fully revealing in the dynamic multi-dimensional cheap talk under Stackelberg equilibria; whereas, the equilibria cannot be fully revealing under a Nash concept. In the dynamic signaling game, affine policies constitute an invariant subspace under best response maps under Nash equilibria. We have provided conditions under which the Stackelberg equilibrium is non-informative through information theoretic arguments. Finally, for dynamic Stackelberg signaling games involving Gauss-Markov sources and memoryless Gaussian channels, we have proved that for scalar setups linear policies are optimal, whereas this is not the case for general multi-dimensional setups.

\section{REFERENCES}

[1] V. P. Crawford and J. Sobel, "Strategic information transmission," Econometrica, vol. 50, pp. 1431-1451, 1982.

[2] T. Başar and G. Olsder, Dynamic Noncooperative Game Theory. Philadelphia, PA: SIAM Classics in Applied Mathematics, 1999.

[3] I. Shames, A. Teixeira, H. Sandberg, and K. Johansson, "Agents misbehaving in a network: a vice or a virtue?" IEEE Network, vol. 26, no. 3, pp. 35-40, May 2012.

[4] S. Sarıtaş, S. Yüksel, and S. Gezici, "Quadratic multi-dimensional signaling games and affine equilibria," IEEE Transactions on Automatic Control, vol. 62, no. 2, pp. 605-619, Feb. 2017.

[5] S. Sarıtaş, S. Yüksel, and S. Gezici, "Dynamic quadratic cheap talk and signaling games," in preparation.

[6] M. Golosov, V. Skreta, A. Tsyvinski, and A. Wilson, "Dynamic strategic information transmission," Journal of Economic Theory, vol. 151, pp. 304-341, 2014.
[7] F. Farokhi, A. M. H. Teixeira, and C. Langbort, "Estimation with strategic sensors," IEEE Transactions on Automatic Control, vol. 62, no. 2, pp. 724-739, Feb. 2017.

[8] E. Akyol, C. Langbort, and T. Başar, "Information-theoretic approach to strategic communication as a hierarchical game," Proceedings of the IEEE, Special Issue on Principles and Applications of Science of Information, vol. 105, no. 2, pp. 205-218, Feb. 2017.

[9] M. O. Sayın, E. Akyol, and T. Başar, "Hierarchical multi-stage Gaussian signaling games," arXiv preprint arXiv:1609.09448, 2016.

[10] S. Sarıtaş, S. Yüksel, and S. Gezici, "Dynamic signaling games under Nash and Stackelberg equilibria," in IEEE International Symposium on Information Theory (ISIT), Barcelona, Spain, July 2016, pp. 16311635.

[11] S. Fabricius, P. Furrer, S. Kerner, T. Linder, and S. Yüksel, "Game theory and information, Queen's University, MTHE 493 Technical Report," Apr. 2014.

[12] I. I. Gihman and A. V. Skorohod, Controlled Stochastic Processes. New York, NY: Springer-Verlag New York, 1979.

[13] V. S. Borkar, "White-noise representations in stochastic realization theory," SIAM J. on Control and Optimization, vol. 31, pp. 1093$1102,1993$.

[14] R. Bansal and T. Başar, "Simultaneous design of measurement and control strategies in stochastic systems with feedback," Automatica, vol. 45, pp. 679-694, Sep. 1989.

[15] S. Yüksel and T. Başar, Stochastic Networked Control Systems: Stabilization and Optimization under Information Constraints. Boston, MA: Birkhäuser, 2013.

[16] C. D. Charalambous, P. A. Stavrou, and N. U. Ahmed, "Nonanticipative rate distortion function and relations to filtering theory," IEEE Transactions on Automatic Control, vol. 59, no. 4, pp. 937-952, Apr. 2014.

[17] M. Gastpar, B. Rimoldi, and M. Vetterli, "To code, or not to code: Lossy source-channel communication revisited," IEEE Transactions on Information Theory, vol. 49, pp. 1147-1158, May 2003.

[18] E. Akyol and K. Rose, "On linear transforms in zero-delay Gaussian source channel coding," in Proceedings of the IEEE International Symposium on Information Theory, Boston, MA, 2012, pp. 15481552.

[19] R. Pilc, "The optimum linear modulator for a Gaussian source used with a Gaussian channel," IEEE Transactions on Automatic Cotrol, vol. 48, pp. 3075-3089, Nov. 1969.

[20] I. Csiszar and J. Korner, Information Theory: Coding Theorems for Discrete Memoryless Channels. Budapest: Akademiai Kiado, 1981.

[21] T. Berger, Rate Distortion Theory. Englewood Cliffs, NJ: PrenticeHall, 1971.

[22] S. Tatikonda and S. Mitter, "Control under communication constraints," IEEE Transactions on Automatic Control, vol. 49, no. 7, pp. 1056-1068, 2004.

[23] S. Tatikonda, A. Sahai, and S. Mitter, "Stochastic linear control over a communication channels," IEEE Transactions on Automatic Control, vol. 49, pp. 1549-1561, Sep. 2004.

[24] K. H. Lee and D. P. Petersen, "Optimal linear coding for vector channels," IEEE Transactions Commun., vol. 24, pp. 1283-1290, 1976.

[25] T. Başar, "A trace minimization problem with applications in joint estimation and control under nonclassical information," J. of Optimization Theory and Applications, vol. 31, pp. 343-359, July 1980.

[26] — - Performance Bounds and Optimal Linear Coding for Multichannel Communication Systems. Bogazici University: PhD Dissertation, 1978.

[27] S. A. Zaidi, T. Oechtering, S. Yüksel, and M. Skoglund, "Stabilization over Gaussian networks," Preprint, 2012.

[28] A. A. Zaidi, T. J. Oechtering, S. Yüksel, and M. Skoglund, "Stabilization and control over Gaussian networks," in Information and Control in Networks, Editors: G. Como, B. Bernhardsson, A. Rantzer. Springer, 2013.

[29] T. Başar and R. Bansal, "Optimum design of measurement channels and control policies for linear-quadratic stochastic systems," European J. Operations Research, vol. 73, pp. 226-236, Dec. 1994. 